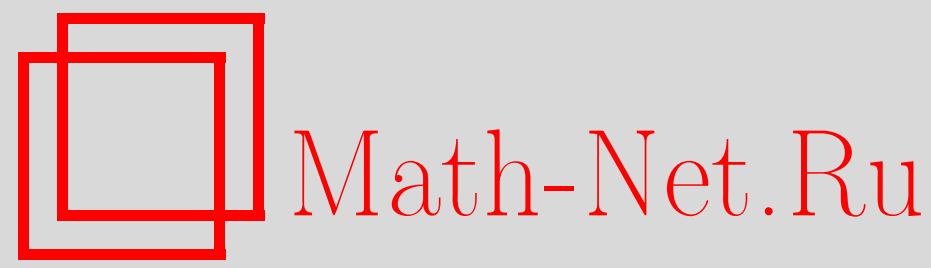

А. Г. Лосев, Об одном критерии гиперболичности некомпактных римановых многообразий специального вида, Матем. заметки, 1996, том 59, выпуск 4, 558-564

DOI: https://doi.org/10.4213/mzm1750

Использование Общероссийского математического портала MathNet.Ru подразумевает, что вы прочитали и согласны с пользовательским соглашением

http://www.mathnet.ru/rus/agreement

Параметры загрузки:

IP : 34.229 .45 .116

26 апреля 2023 г., 13:07:47

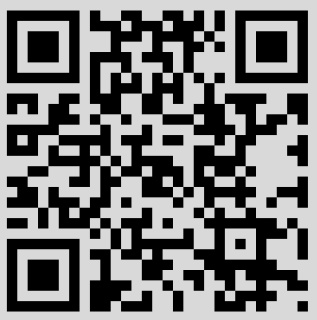




\section{ОБ ОДНОМ КРИТЕРИИ ГИПЕРБОЛИЧНОСТИ НЕКОМПАКТНЫХ РИМАНОВЫХ МНОГООБРАЗИЙ СПЕЦИАЛЬНОГО ВИДА}

\section{А. Г. Лосев}

Данная статья посвящена изучению поведения ограниченных гармонических функций на полньх римановых многообразиях некоторого специального вида в зависимости от геометрии многообразия.

Классическая теорема Лиувилля утверждает, что всякая ограниченная гармоническая функция в $\mathbb{R}^{n}$ является константой. Однако класс многообразий, на которых существуют нетривиальные ограниченные гармонические функции, достаточно обширен. Так, например, М. Андерсоном [1] и Д. Сулливаном [2] была решена задача Дирихле для полного односвязного многообразия с секционной кривизной, ограниченной двумя отрицательными константами. В развитие данного вопроса С. Т. Яу [3] предложил следующую классификацию римановых многообразий.

ОПРЕДЕЛЕНИЕ. Будем называть многообразие строго параболическим, если всякая положительная гармоническая функция на нем равна константе.

Многообразие называют параболическим, если множество положительных гармонических функций на нем имеет конечную размерность.

Остальные многообразия являются әиперболическими, т.е. непараболическими.

В данной работе приведен критерий гиперболичности полных римановых многообразий некоторого специального вида.

Пусть $\mathscr{M}$ - полное риманово многообразие, представимое в виде объединения $\mathscr{M}=\mathscr{B} \cup \mathscr{D}$, где $\mathscr{B}$ - некоторьй компакт, а $\mathscr{D}$ изометрично прямому произведению $\mathbb{R}_{+} \times \mathscr{S}$ (где $\mathbb{R}_{+}=(0, \infty)$, а $\mathscr{S}$ - компактное риманово многообразие) с метрикой

$$
d s^{2}=h^{2}(r) d r^{2}+g^{2}(r) d \theta^{2} .
$$

Здесь $h(r)$ и $g(r)$ - положительные, гладкие на $\mathbb{R}_{+}$функции, а $d \theta^{2}$ - метрика на $\mathscr{S}$. Примерами таких многообразий могут служить евклидово

(C) А. Г. Лосев 1996 
пространство $(h(r)=1, g(r)=r)$, пространство Лобачевского $(h(r)=1$, $g(r)=\operatorname{sh}(r))$, поверхность, полученная вращением графика функции $f(r)$ вокруг луча $O r$ в $\mathbb{R}^{n}\left(h(r)=\sqrt{1+\left|f^{\prime}(r)\right|^{2}}, g(r)=f(r)\right)$ и другие.

Введем обозначение

$$
I=\int_{r_{0}}^{\infty} h(t) g^{1-n}(t)\left(\int_{r_{0}}^{t} h(\xi) g^{n-3}(\xi) d \xi\right) d t
$$

где $r_{0}=$ const $>0, n=\operatorname{dim} \mathscr{M}$.

Справедливо следующее утверждение.

Теорема. 1) Пусть риманово многообразие $\mathscr{M}$ таково, ито $I=\infty$. Тогда оно является строго параболическим.

2) Пусть риманово многообразие $\mathscr{M}$ таково, что $I<\infty$. Тогда оно является гиперболическим. Более того, для любой непрерывной на $\mathscr{S}$ функиии $\Phi(\theta)$ существует гармоническая функиия и такая, что

$$
\lim _{r \rightarrow \infty} u(r, \theta)=\Phi(\theta) .
$$

ЗАмЕчАнИЕ 1 . Из условия $I<\infty$ следует, что

$$
\int_{r_{0}}^{\infty} h(t) g^{1-n}(t) d t<\infty
$$

Последнее эквивалентно существованию функции Грина многообразия $\mathscr{M}$ (см. [4]).

ДОКАЗАТЕЛЬСТВО ТЕОРЕмЫ. Прежде всего заметим, что первое утверждение теоремы доказано в [4]. Докажем второе утверждение теоремы. Заметим вначале, что в координатах $(r, \theta)$ оператор Лапласа-Бельтрами на $\mathscr{D}$ имеет вид

$$
\Delta=\frac{1}{h^{2}(r)} \cdot \frac{\partial^{2}}{\partial r^{2}}+\frac{1}{h^{2}(r)}\left((n-1) \frac{g^{\prime}(r)}{g(r)}-\frac{h^{\prime}(r)}{h(r)}\right) \frac{\partial}{\partial r}+\frac{1}{g^{2}(r)} \Delta_{\theta},
$$

где $\Delta_{\theta}$ - внутренний лапласиан на $\mathscr{S}$. Формула проверяется непосредственно по определению оператора Лапласа-Бельтрами (см. также [4]).

Пусть $w_{k}$ - ортонормированньй базис в $L^{2}(\mathscr{S})$ из собственных функций оператора Лапласа $\Delta_{\theta}$, а $\lambda_{k}$ - соответствуюшие собственные числа. Тогда для любого $r$ имеем

$$
u(r, \theta)=\sum_{k=0}^{\infty} v_{k}(r) w_{k}(\theta)
$$


где

$$
v_{k}(r)=\int_{\mathscr{S}} u(r, \theta) w_{k}(\theta) d \theta, \quad \Delta_{\theta} w_{k}(\theta)+\lambda_{k} w_{k}(\theta)=0 .
$$

Из (1) следует, что для любого номера $k$ функция $v_{k}(r)$ является решением следующего обыкновенного дифференциального уравнения

$$
v^{\prime \prime}(r)+\left((n-1) \frac{g^{\prime}(r)}{g(r)}-\frac{h^{\prime}(r)}{h(r)}\right) v^{\prime}(r)-\lambda \frac{h^{2}(r)}{g^{2}(r)} v(r)=0,
$$

где $\lambda=\lambda_{k}$.

Последнее эквивалентно уравнению

$$
\left(\frac{g^{n-1}(r)}{h(r)} v^{\prime}(r)\right)^{\prime}=\lambda g^{n-3}(r) h(r) v(r) .
$$

Проинтегрируем данное уравнение от $r_{0}$ до $r$. В результате получим

$$
v^{\prime}(r)=\lambda \frac{h(r)}{g^{n-1}(r)} \int_{r_{0}}^{r} g^{n-3}(\xi) h(\xi) v(\xi) d \xi+\frac{v^{\prime}\left(r_{0}\right) g^{n-1}\left(r_{0}\right)}{h\left(r_{0}\right)} \cdot \frac{h(r)}{g^{n-1}(r)} .
$$

Интегрируя еще раз от $r_{0}$ до $r$, получаем

$$
\begin{aligned}
v(r)= & \lambda \int_{r_{0}}^{r} \frac{h(t)}{g^{n-1}(t)}\left(\int_{r_{0}}^{t} g^{n-3}(\xi) h(\xi) v(\xi) d \xi\right) d t \\
& +\frac{v^{\prime}\left(r_{0}\right) g^{n-1}\left(r_{0}\right)}{h\left(r_{0}\right)} \int_{r_{0}}^{r} \frac{h(t)}{g^{n-1}(t)} d t+v\left(r_{0}\right) .
\end{aligned}
$$

Переобозначим решение уравнения (2) $v(r)$ с $\lambda=\lambda_{k}$ и граничньми условиями $v\left(r_{0}\right)=1, v^{\prime}\left(r_{0}\right)=0$ через $l_{k}(r)$. Тогда из (3) очевидно следует, что $l_{k}(r)$ - монотонно возрастающая, положительная функция.

Покажем теперь ограниченность $l_{k}(r)$ на $\left(r_{0},+\infty\right)$. Так как $l_{k}(r)$ монотонно возрастает на $\left(r_{0},+\infty\right)$ и положительна, то из (3) следует, что

$$
l_{k}^{\prime}(r) \leqslant \lambda_{k} g^{1-n}(r) h(r) l_{k}(r) \int_{r_{0}}^{r} h(\xi) g^{n-3}(\xi) d \xi .
$$

Отсюда

$$
\left(\ln l_{k}(r)\right)^{\prime} \leqslant \lambda_{k} \frac{h(r)}{g^{n-1}(r)} \int_{r_{0}}^{r} h(\xi) g^{n-3}(\xi) d \xi .
$$

Интегрируя данное неравенство от $r_{0}$ до $r$, получаем

$$
l_{k}(r) \leqslant \exp \left(\lambda_{k} \int_{r_{0}}^{r} h(t) g^{1-n}(t)\left(\int_{r_{0}}^{t} g^{n-3}(\xi) h(\xi) d \xi\right) d t\right) .
$$


Из условия $I<\infty$ получаем ограниченность $l_{k}(r)$.

Из монотонного возрастания и ограниченности функции $l_{k}(r)$ следует существование предела

$$
\lim _{r \rightarrow \infty} l_{k}(r)=b_{k}
$$

для всех $k=0,1,2, \ldots$.

Заметим, далее, что для любого числа $c_{k}$ функция

$$
v_{k}(r)=\frac{c_{k}}{b_{k}} l_{k}(r)
$$

является решением уравнения (1) и

$$
\lim _{r \rightarrow \infty} v_{k}(r)=c_{k}
$$

Кроме того, очевидно, $\left|v_{k}(r)\right|$ - монотонно возрастающая функция.

Далее докажем, что ряд

$$
\sum_{k=0}^{\infty} v_{k}(r) w_{k}(\theta)
$$

равномерно сходится на $\left(r_{0},+\infty\right)$.

Пусть $\Phi(\theta)$ - бесконечно дифференцируемая функция на $\mathscr{S}$. Заметим, что

$$
\Phi(\theta)=\sum_{k=0}^{\infty} c_{k} w_{k}(\theta)
$$

где

$$
c_{k}=\int_{\mathscr{S}} \Phi(\theta) w_{k}(\theta) d \theta
$$

а $w_{k}(\theta)$ определена вьше.

Используя формулу Грина и определение $w_{k}(\theta)$, для $k \neq 0$ получаем

$$
\begin{aligned}
\left|c_{k}\right| & =\left|\int_{\mathscr{S}} \Phi(\theta) w_{k}(\theta) d \theta\right|=\frac{1}{\lambda_{k}}\left|\int_{\mathscr{S}} \Phi(\theta) \Delta_{\theta} w_{k}(\theta) d \theta\right| \\
& =\frac{1}{\lambda_{k}}\left|\int_{\mathscr{S}} \Delta_{\theta} \Phi(\theta) w_{k}(\theta) d \theta\right| .
\end{aligned}
$$

Применяя формулу Грина $p$ раз (где $p$ - произвольноенатуральное число), получим

$$
\left|c_{k}\right|=\frac{1}{\lambda_{k}^{p}}\left|\int_{\mathscr{S}} \Delta_{\theta}^{p} \Phi(\theta) w_{k}(\theta) d \theta\right| .
$$


Применяя к правой части неравенство Коши-Буняковского, имеем

$$
\left|c_{k}\right| \leqslant \frac{1}{\lambda_{k}^{p}} \sqrt{\int_{\mathscr{S}}\left(\Delta_{\theta}^{p} \Phi(\theta)\right)^{2} d \theta} \sqrt{\int_{\mathscr{S}} w_{k}^{2}(\theta) d \theta}=\frac{a}{\lambda_{k}^{p}},
$$

где $a-$ константа, зависящая от $\mathscr{S}$ и $p$. Из асимптотики Вейля следует, что $\lambda_{k} \geqslant a_{1} k^{2 / n}$, где $a_{1}-$ константа, зависящая от $\mathscr{S}$. Поэтому

$$
\left|c_{k}\right| \leqslant \frac{a}{a_{1}^{p} k^{2 p / n}} .
$$

Из теоремы вложения Соболева и стандартных эллиптических оценок вытекает

$$
\left|w_{k}(\theta)\right|<a_{2} k^{N}
$$

где $a_{2}$ и $N$ - константы, зависяшие от компакта $\mathscr{S}$. Тогда справедливы следуюшие неравенства

$$
\begin{aligned}
\left|\sum_{k=1}^{\infty} v_{k}(r) w_{k}(\theta)\right| & \leqslant \sum_{k=1}^{\infty}\left|v_{k}(r)\right| \cdot\left|w_{k}(\theta)\right| \leqslant \sum_{k=1}^{\infty}\left|c_{k}\right| \cdot\left|w_{k}(\theta)\right| \\
& \leqslant \frac{a a_{2}}{a_{1}^{p}} \sum_{k=1}^{\infty} k^{N-2 p / n}
\end{aligned}
$$

и при достаточно большом $p$ ряд сходится. Следовательно, ряд

$$
\sum_{k=0}^{\infty} v_{k}(r) w_{k}(\theta)
$$

сходится абсолютно и равномерно.

Учитывая, что

$$
\lim _{r \rightarrow \infty} v_{k}(r)=c_{k}
$$

получаем

$$
\lim _{r \rightarrow \infty} \sum_{k=0}^{\infty} v_{k}(r) w_{k}(\theta)=\sum_{k=0}^{\infty} c_{k} w_{k}(\theta)=\Phi(\theta) .
$$

Таким образом, мы построили на $\mathscr{M} \backslash \mathscr{B}$ гармоническую функцию

$$
u_{0}(r, \theta)=\sum_{k=0}^{\infty} v_{k}(r) w_{k}(\theta),
$$

удовлетворяющую условиям

$$
\lim _{r \rightarrow \infty} u_{0}(r, \theta)=\Phi(\theta),\left.\quad \frac{\partial u_{0}}{\partial \nu}\right|_{\partial \mathscr{B}}=0
$$


(последнее следует из $v^{\prime}\left(r_{0}\right)=0$ ).

Построим гармоническую на всем многообразии $\mathscr{M}$ функцию $u(x)$ такую, что

$$
\lim _{r \rightarrow \infty}\left(u(r, \theta)-u_{0}(r, \theta)\right)=0 .
$$

Для этого рассмотрим последовательность функций, удовлетворяющих задаче

$$
\Delta \varphi_{i}=0 \quad \text { в } B_{i},\left.\quad \varphi_{i}\right|_{\partial B_{i}}=\left.u_{0}\right|_{\partial B_{i}},
$$

где $B_{i}$ - возрастающая последовательность предкомпактных подмножеств $\mathscr{M}$, имеющих гладкие границы и исчерпывающих $\mathscr{M}$.

Пусть $G_{i}(x, y)$ - функция Грина предкомпакта $B_{i}$. Кроме того, пусть $\varphi_{0}$ - финитная функция, равная нулю в $\mathscr{B}$ и равная единице вне некоторой окрестности $\mathscr{B}$. Тогда положим $U=u_{0} \varphi_{0}, \Delta U=f$. Заметим, что $\operatorname{supp} f$ лежит в некоторой окрестности $\mathscr{B}$. Рассмотрим последовательность функций $\psi_{i}=\varphi_{i}-U$. Для них выполнено $\Delta \psi_{i}=-f,\left.\psi_{i}\right|_{\partial B_{i}}=0$. Тогда

$$
\psi_{i}(x)=\int_{B_{i}} G_{i}(x, y) f(y) d y .
$$

Из существования предела функций Грина $G_{i}$ при $i \rightarrow \infty$ (см. замечание 1$)$ следует существование предела последовательности $\left\{\psi_{i}\right\}$ и, соответственно, сушествование предела последовательности $\left\{\varphi_{i}\right\}$.

Обозначим

$$
\lim _{i \rightarrow \infty} \varphi_{i}=u,
$$

где $u$ - гармоническая функция. Кроме того, справедливы равенства

$$
\begin{aligned}
\int_{\partial B_{i}} \frac{\partial \psi_{i}}{\partial \nu} & =\int_{B_{i}} \Delta \psi_{i}=-\int_{B_{i}} f=-\int_{B_{i}} \Delta\left(u_{0} \varphi_{0}\right) \\
& =-\int_{\partial B_{i}} \frac{\partial u_{0}}{\partial \nu}=-\int_{\partial B_{i} \cup \partial \mathscr{B}} \frac{\partial u_{0}}{\partial \nu}=-\int_{B_{i} \backslash \mathscr{B}} \Delta u_{0}=0 .
\end{aligned}
$$

Отсюда получаем (см. также [4]), что для любой предкомпактной области $K$ вьполнено

$$
\sup _{\mathscr{M} \backslash K}\left(u-u_{0}\right)=\sup _{\partial K}\left(u-u_{0}\right), \quad \inf _{\mathscr{M} \backslash K}\left(u-u_{0}\right)=\inf _{\partial K}\left(u-u_{0}\right) .
$$

Учитывая, что

$$
\lim _{r \rightarrow \infty} u_{0}=\Phi(\theta)
$$

получаем

$$
\lim _{r \rightarrow \infty} u=\Phi(\theta)
$$


Таким образом, на многообразии $\mathscr{M}$ решена задача Дирихле для любой бесконечно дифференцируемой функции $\Phi(\theta)$.

Пусть теперь на $\mathscr{S}$ задана непрерьвная функция $\Phi(\theta)$. И пусть $\left\{\Phi_{i}\right\}-$ последовательность бесконечно дифференцируемых функций, равномерно сходящаяся к $\Phi$. Пусть $\left\{u_{i}\right\}$ - последовательность решений задачи Дирихле на $\mathscr{M}$ с граничными функциями $\Phi_{i}$. Тогда функция

$$
u=\lim _{i \rightarrow \infty} u_{i}
$$

будет решением задачи Дирихле с граничной функцией $\Phi(\theta)$. Теорема доказана.

ЗАмЕЧАниЕ 2. Пусть $\mathscr{M}$ - полное риманово многообразие, устроенное следуюшим образом: внешность некоторого компакта $\mathscr{B}$ в $\mathscr{M}$ состоит из $m$ компонент связности $D_{1}, \ldots, D_{m}$, каждая из которых изометрична прямому произведению $\mathbb{R}_{+} \times \mathscr{S}$ с метрикой

$$
d s^{2}=h_{i}^{2}(r) d r^{2}+g_{i}^{2}(r) d \theta^{2} .
$$

Введем обозначение

$$
I_{i}=\int_{r_{0}}^{\infty} h_{i}(t) g_{i}^{1-n}(t)\left(\int_{r_{0}}^{t} h_{i}(\xi) g_{i}^{n-3}(\xi) d \xi\right) d t
$$

Пусть $\mathscr{M}$ таково, что все $I_{i}=\infty$ и по крайней мере один из интегралов

$$
\int_{r_{0}}^{\infty} h_{i}(t) g_{i}^{1-n}(t) d t<\infty
$$

В работе [4] показано, что размерность множества положительных гармонических функций на таких многообразиях равно $m$. Таким образом, при $m>1$ так устроенные многообразия являются многообразиями параболического типа.

Волгоградский государственный университет Поступило

E-mail: losev@math.vgu.tsaritsyn.su

\section{СПИСОК ЦИТИРОВАННОЙ ЛИТЕРАТУРЫ}

[1] Anderson M. T. The Dirichlet problem at infinity for manyfolds with negative curvature // J. Diff. Geom. 1983. V. 18. P. 701-722.

[2] Sullivan D. The Dirichlet problem at infinity for a negatively curved manyfolds // J. Diff. Geom. 1983. V. 18. P. 723-732.

[3] Yau S. T. Nonlinear analysis in geometry // L'Enseigenement Mathematique. 1987. V. 33. P. 109-158.

[4] Лосев А. Г. Некоторые лиувиллевы теоремы на римановых многообразиях специального вида // Изв. вузов. Матем. 1991. № 12. С. 15-24. 